\title{
O IMPACTO DAS ATIVIDADES INOVATIVAS NA AMPLIAÇÃO DE MERCADO NOS SETORES INDUSTRIAIS BRASILEIROS
}

Rodrigo Milano de Lucena

\section{Resumo}

O presente buscou relacionar o impacto da ampliação de mercado dos setores industriais brasileiros com as atividades inovativas de dispêndios de inovação. Para tanto, utilizou-se de dados das PINTEC fornecidos pelo IBGE para os anos de 2000, 2003, 2005, 2008 e 2011. Como método de análise utilizou-se a econometria de dados em painel e modelagem de regressões de efeitos fixos e efeitos aleatórios. A conclusão é de que com exceção dos dispêndios com Pesquisa e Desenvolvimento Interno a empresa, os demais gastos com inovação apresentam impactos positivos e confiáveis estatisticamente na ampliação de mercado dos setores industriais brasileiros.

\section{Introdução}

Na literatura, a inovação é considerada como variável-chave para o desenvolvimento do país conforme defende Sbragia (2006). Essa importância se deve aos reflexos da inovação na competitividade da indústria e o desenvolvimento da sociedade em todas as dimensões (Dosi, 2000).

As questões que envolvem a temática da inovação hoje dizem respeito à estrutura, dinâmica, mecanismos de quantificação e valoração, modelos de comparação, avaliação de eficácia, entre outros (Tidd, Bessant e Pavitt, 2008).

Os diferentes resultados das pesquisas empíricas sobre o impacto da inovação vêm impulsionando outros estudos para um melhor entendimento das causas e efeitos da inovação. Porém mesmo com esses esforços a heterogeneidade de variáveis, as dificuldades em compreender as relações e distinguir as ambiguidades têm dificultado a análise de pesquisas nesta área (Cainelli, Evanglista e Savona, 2004).

Diante disso, presente trabalho tem como objetivo contribuir para a investigação da temática da inovação das empresas estudando o impacto que os dispêndios relacionados à inovação exercem sobre a ampliação do poder de mercado dos setores industriais brasileiros. Para isso utilizou dados da PINTEC - Pesquisa de Inovação Tecnológica do IBGE, dos anos de 2000, 2003, 2005, 2008 e 2011. 
Esse trabalho encontra-se organizado em 4 seções assim distribuídas além dessa introdução: revisão de literatura, procedimentos metodológicos, resultados e discussões e conclusão.

\section{Revisão de Literatura}

As empresas buscam através da inovação de produtos e processos, defender um posicionamento competitivo em seus respectivos mercados bem como crescimento econômico (DOSI e NELSON, 1994). Para que esse objetivo seja alcançado, é preciso que as corporações incluam a inovação como pauta de seu planejamento estratégico para que os recursos sejam direcionados com efetividade para os esforços inovativos (FREEMAN e SOETE, 2008), já que tal como se observa em estatísticas mundiais acerca dos aportes de em P\&D\&I, os investimentos privados são fundamentais para o desenvolvimento científico e tecnológico.

Na Coreia, por exemplo, o setor privado foi responsável por $84 \%$ do total de investimentos em pesquisa até 1994 evidenciando que as empresas buscaram competitividade em setores estratégicos da economia. Contudo, os financiamentos preferenciais e as concessões tributárias foram instrumentos eficazes na promoção do crescimento de $\mathrm{P} \& \mathrm{D}$ das empresas coreanas. Esses investimentos permitiram a redução de custos de P\&D e de desenvolvimento de recursos humanos das empresas (KIM, 1997), o que justifica o fato de que as empresas necessitam de estímulos externos para investirem em inovação. Ou seja, mesmo que as empresas foram as responsáveis pelo avanço tecnológico coreano, o papel do Estado como adequação do ambiente institucional para a inovação foi relevante.

Porém no Brasil, o esforço de dispêndios de P\&D das empresas brasileiras representa apenas 4\% do total dos investimentos de pesquisa, deixando para o Estado a responsabilidade de arcar com esses dispêndios, além de se esforçar, mesmo que minimamente, na criação de um ambiente propício para a inovação. Mesmo com políticas industriais e de inovação brasileiras, as empresas ainda não se encontram motivadas a estimular P\&D no interior da indústria, buscando formas de apropriar de produtos inovadores a partir de parcerias externas ao ambiente produtivo.

Mesmo quando as empresas inovam, essas inovações se concentram nas inovações incrementais que denotam melhoria na qualidade de produtos e processos empresariais, porém não criam novos produtos e processos o que geralmente não gera o surgimento de novos mercados (SANTOS E NEVES, 2013).

Outro fator relevante para o fato de não haver inovação dentro da indústria brasileira está no fato de que $72 \%$ dedicados à pesquisa estão nas universidades e por isso a 
aproximação desses profissionais com a cadeia produtiva ainda é distante (SBRAGIA, 2006).

Rocha (2015), argumenta que o esforço governamental à inovação das empresas do setor de transformação não afetou positivamente nos gastos de P\&D da iniciativa privada, sugerindo assim, um erro no foco da política de inovação brasileira e que uma postura mais associada à visão sistêmica da inovação deve ser adotada.

Um fato importante que motiva a indústria a inovar é a competitividade no mercado. Empresas buscam competitividade a fim de dominar uma tecnologia e explorar o mercado tentando deixar seus concorrentes fora da competição. Portanto, a ampliação de mercado das empresas torna-se uma variável-chave para estimular a inovação.

Porém a decisão de incorporar a estratégia da inovação no planejamento das organizações não é um processo comum já que isso requer grandes esforços administrativos e os resultados são incertos e quando acontecem, geralmente são obtidos no longo prazo (DOSI, 2000).

Assume-se, portanto a ampliação de mercado das empresas industriais como meta das organizações a fim de provocar mudanças nas estruturas de mercado e obter crescimento econômico e diversas variáveis são relevantes no alcance desse objetivo, tais como tamanho do mercado consumidor, número de concorrentes, financeirização da economia, dentre outras variáveis. A fim de contribuir na temática de estudo, o trabalho incorpora os gastos com atividades inovativas como variáveis explicativas na ampliação do poder de mercado.

\section{Procedimentos metodológicos}

A fonte de dados utilizada no trabalho foram as PINTEC disponibilizadas pelo IBGE para os anos de 2000, 2003, 2005, 2008 e 2011. A PINTEC possui dois grandes blocos de variáveis a saber: Atividades inovativas e Impacto das Inovações.

O trabalho buscou explicar qual é a relação do impacto da ampliação de mercado dos setores industriais (um dos impactos) com os dispêndios de inovação (um dos conjuntos das atividades inovativas). Dentre as variáveis que a pesquisa fornece, foi selecionado o impacto causado na ampliação da participação da empresa no mercado como variável dependente, já que esse é um dos objetivos de se investir em inovação.

O impacto é mensurado em três níveis: alto, médio e baixo ou não relevante. O objetivo da pesquisa buscou verificar a ampliação do impacto considerado “alto” pelas atividades inovativas. Para que a análise pudesse ser feita necessitou construir a variável através da proporção do impacto alto sobre o total de impacto que foi percebido pelos setores industriais, criando assim a variável que foi denominada "Mercado" no modelo estimado.

No caso dos dispêndios de inovação, a construção das variáveis se deu através do total 
de dispêndio que o setor industrial teve no período divido pela receita total do setor industrial, no período analisado.

Os dispêndios de inovação disponíveis na PINTEC que foram utilizados no trabalho foram: Pesquisa de Desenvolvimento Interno a Empresa (PDI), Pesquisa e Desenvolvimento Externo a Empresa (PDE), Conhecimento Externo (CE), Treinamentos (TT). Essas são as variáveis exógenas do modelo.

Trabalhou-se nessa pesquisa com 35 setores industriais cujos dados estavam disponíveis em todas as 5 edições e a nomenclatura foi constante a fim de tornar o painel o mais balanceado possível.

Vale ressaltar que a partir de 2006 uma nova Classificação Nacional de Atividade Econômica foi adotada, a chamada CNAE 2.0. No trabalho o autor utilizou dados da CNAE 1.0 disponíveis para os anos de 2000, 2003, 2005 e 2008. Como no ano de 2011 a pesquisa apresentou somente os dados da CNAE 2.0 utilizou-se a tabela de conversão da CNAE 2.0 para 1.0 disponível na Comissão Nacional de Classificação do IBGE a fim de manter a conformidade da base de dados numa perspectiva histórica (ano a ano).

Como o interesse da pesquisa é analisar o impacto dos dispêndios de inovação na indústria nacional, optou-se pelo uso da análise de regressão. Como os dados da PINTEC integram um corte transversal para cada setor durante os 5 anos pesquisados, a técnica multivariada mais adequada para atingir o objetivo encontrada foi a regressão com dados em painel, de forma a construir uma matriz (NxT), onde há observações para 35 setores $(\mathrm{N})$ em 5 períodos (T), o que permite um painel com 175 observações.

A equação de regressão foi modelada a partir da fórmula:

$$
\mathrm{Y}_{\mathrm{it}}=\alpha_{\mathrm{i}}+\beta_{1} \mathrm{PDI}_{\mathrm{it}}+\beta_{2} \mathrm{PDE}_{\mathrm{it}}+\beta_{3} \mathrm{CE}_{\mathrm{it}}+\beta_{4} \mathrm{TT}_{\mathrm{it}}+\varepsilon_{\mathrm{it}}
$$

Os subscritos “i” e " $\mathrm{t}$ ” representam os setores individuais em cada período de tempo, respectivamente. Os betas $(\beta)$ são os coeficientes angulares de cada variável independente. A variável dependente (Mercado) assumirá a posição de Y na equação.

\section{Resultados e discussões}

Não foi necessária a transformação logarítmica das variáveis uma vez as plotagens gráficas dos valores não alteraram significativamente a relação entre as variáveis independentes e a variável dependente e após testes de inclusão e exclusão de variáveis no modelo chegou-se ao resultado da regressão apresentado na tabela 1 abaixo: 
Fonte: elaborado pelo autor a partir de resultados da pesquisa

Através de testes estatísticos verificou-se que tanto o histograma quanto o gráfico normal quantílico dos erros do modelo ajustado se aproximam de uma distribuição normal, apesar de o histograma estar levemente assimétrico a esquerda, conforme Figura 1.
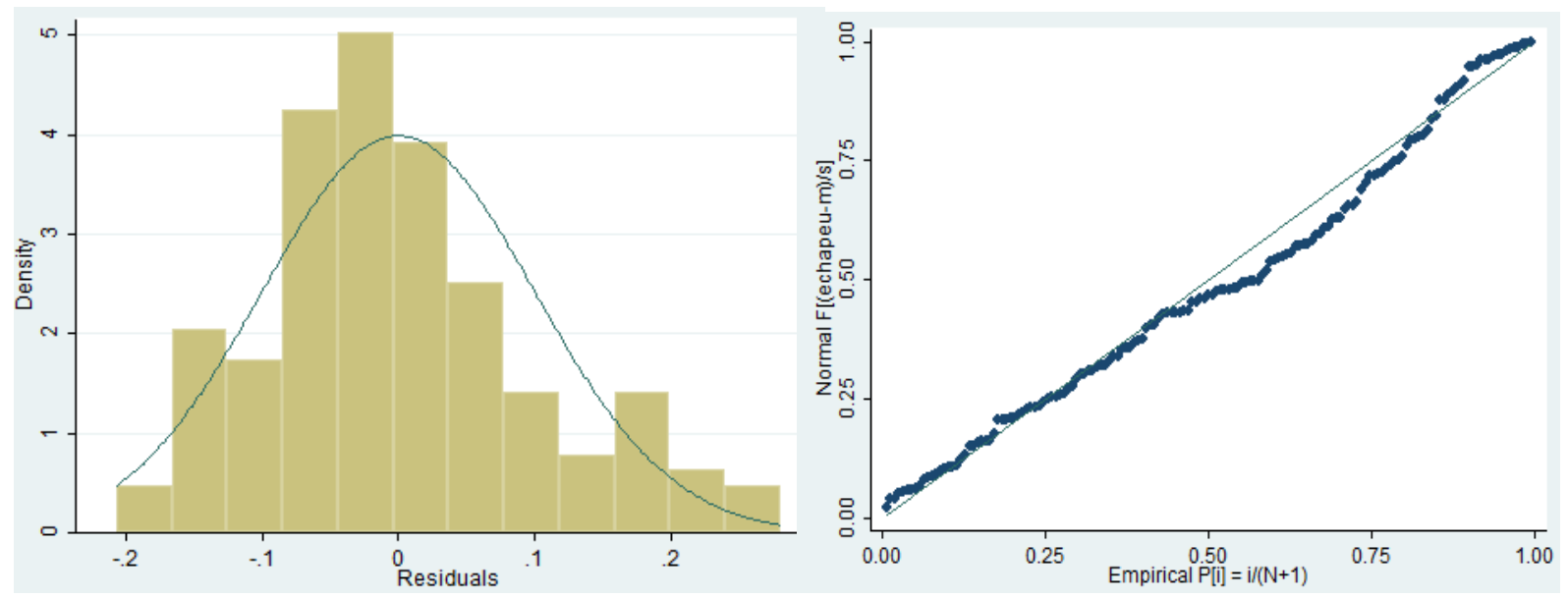

Figura1: Histograma e gráfico quantílico da distribuição dos erros do modelo estimado

Após a análise da distribuição normal dos erros é necessário também verificar a presença de multicolinearidade no modelo estimado e para tanto utilizou o comando VIF do Stata para verificar o fator inflacionário da variância e determinar a presença ou não de multicolinearidade. A Tabela 2 apresenta o resultado do teste: 
Verifica-se que duas das variáveis estão próximos do valor 1, ou seja, são quase que independentes no modelo. As variáveis PDI e PDE possuem valores maiores, porém não acima de 5 e como essas variáveis são importantes de acordo com a literatura da inovação, decidiu-se por manter tais variáveis já que os valores do teste não foram maiores do que 10, onde indicariam nesse caso de cautela especial para os efeitos dessa multicolinearidade.

O problema mais frequente em dados em painel é a questão da heterogeneidade não observada. Nesse caso, haveria fatores que determinam a variável dependente, mas não estão sendo considerados na equação dentro do conjunto das variáveis explicativas por não serem diretamente observáveis. Portanto o modelo pode conter um coeficiente $\mathrm{c}_{\mathrm{i}}$ em cada unidade observacional constante ao longo do tempo.

Se o coeficiente $c_{i}$ for correlacionado com qualquer variável do modelo e tentarmos aplicar o MQO as estimativas serão viesadas e inconsistentes. Nesse caso, para que possamos estimar a equação do modelo consistentemente, a abordagem mais usual no contexto de dados longitudinais é o de Efeitos Fixos. Entretanto se esses efeitos forem estritamente nãocorrelacionados com variáveis explicativas, pode ser mais apropriado modelar esses efeitos como sendo aleatoriamente distribuídos entre as unidades observacionais, utilizando o modelo de Efeitos Aleatórios.

Uma solução para controlar a heterogeneidade individual é elaborar um modelo de efeitos fixos com variáveis binárias onde se pressupõe que $c_{i}$ represente parâmetros da população a serem estimados. O modelo evidenciou que se pode afirmar que há diferenças significativas entre os indivíduos já que o valor da estatística t individuais não permitiram rejeitar a hipótese nula de que há diferenças entre o primeiro indivíduo de referência com os demais, com exceção de 3 indivíduos, dos 35 testados. Isso quer dizer que com relação a ampliação de mercado, os setores industriais não se comportam de maneira uniforme.

Como o modelo com a inclusão de binárias consomem muitos graus de liberdade, pode-se elaborar um modelo within com efeitos fixos e o resultado é evidenciado na tabela 3 abaixo: 
Fonte: Elaborado pelo autor a partir de resultados da pesquisa

Verifica-se que a capacidade de explicação da variabilidade pelo $\mathrm{R}^{2}$ within é o maior com cerca de 12,25\% de explicação. O modelo como um todo é significativo já que o teste $\mathrm{F}(4,136)=4,75$ e Prob. $>\mathrm{F}=0,0013$. Porém verifica-se que cerca de $19 \%$ da variabilidade total dos resíduos se deve às diferenças entre as unidades de corte transversal (rho=0,1878).

Seguindo com a verificação de modelos fixos para melhor ajustamento do modelo estimado é necessário verificar também um modelo fixo two-way que considera que o intercepto possa variar entre as unidades de corte transversal (indivíduos) e entre os períodos. O resultado da análise é de que entre não há diferenças entre o ano 2000 (referência) e os anos de 2005 e 2008.

No ajuste do modelo, há também a hipótese de que o intercepto de diferenças entre os indivíduos seja uma variável aleatória e não uma constante, ou seja, as variações individuais seriam identificadas por oscilações aleatórias em torno de uma constante. Esse modelo estimado é chamado de modelo de efeitos aleatórios ou também de modelo de correção de erros. Através do comando xtreg do Stata estimou-se um modelo aleatório one-way considerando que as diferenças individuais ficam em torno de uma constante e é apresentado na tabela 4 abaixo: 
A interpretação dos modelos aleatórios é semelhante ao dos efeitos fixos, ou seja, pelos coeficientes não se mudam os preceitos teóricos. Porém, a variabilidade total dos resíduos não é explicada pelas diferenças aleatórias entre as unidades de corte transversal.

Num modelo de efeitos fixos two-way, é considerado que além das variações aleatórias individuais em torno de uma constante, haja ainda variações aleatórias entre os períodos em torno de uma constante. Em outras palavras, o intercepto pode ser decomposto em duas variáveis aleatórias.

Para ajustar o modelo de efeitos aleatórios two-way, utilizou-se do comando xtmixed do Stata e o resultado é apresentado na Tabela 5 abaixo:

\begin{tabular}{|c|c|c|c|c|c|c|}
\hline \multicolumn{3}{|c|}{ log likehood = 174.93834} & \multicolumn{4}{|c|}{$\begin{array}{l}\text { Wald chi } 2(4)=28.46 \\
\text { Prob }>\text { chi2 }=0.0000\end{array}$} \\
\hline Mercado & Coef. & $\begin{array}{l}\text { Std. } \\
\text { Error }\end{array}$ & $\mathrm{Z}$ & $\mathrm{p}>|\mathrm{z}|$ & {$[95 \%$ cor } & f. Interval] \\
\hline PDI & $\begin{array}{l}- \\
04\end{array}$ & 100 & -342 & 1 & & 0.1755158 \\
\hline PDE & 1,340190 & 0,450617 & 3,15 & 0,002 & 0,507313 & 2,1735240 \\
\hline CE & 2,696849 & 0,766602 & 3,52 & 0,000 & 1,194336 & 4,1993620 \\
\hline TT & 1,925081 & 0,711418 & 2,71 & 0,007 & 0,530716 & 3,3194350 \\
\hline _cons & 0,3444795 & 0,026424 & 13,04 & 0,000000 & 0,292689 & 0,3962702 \\
\hline
\end{tabular}

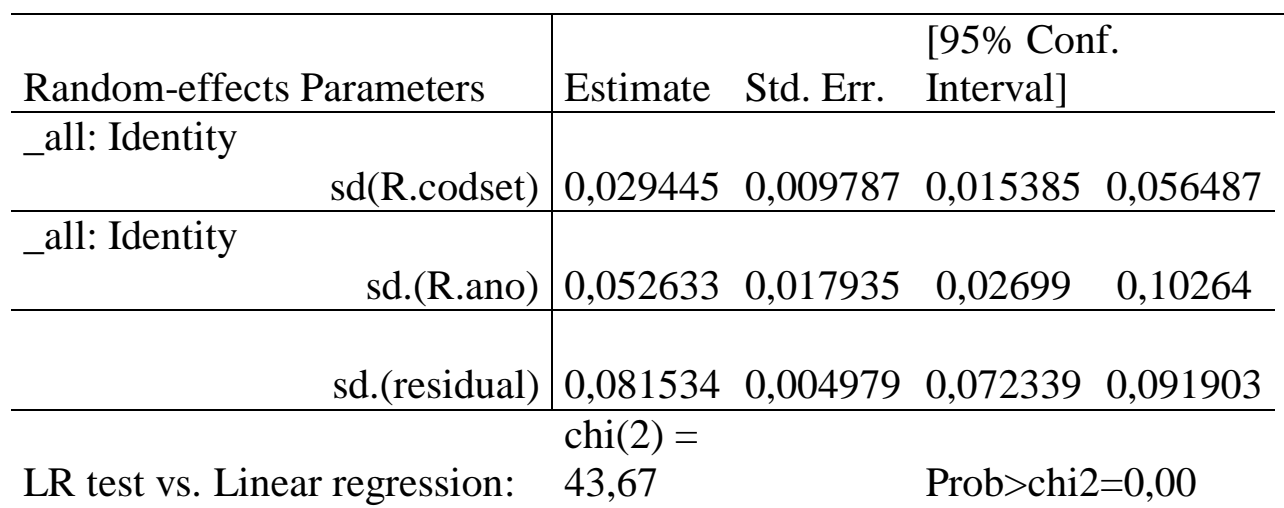

Fonte: elaborado pelo autor a partir de dados da pesquisa

O resultado abaixo parte do pressuposto de que as variações entre os setores industriais e os anos estejam normalmente distribuídos em torno de uma constante. Também é pressuposto correlação entre os erros compostos tanto entre indivíduos diferentes de um mesmo período como entre períodos diferentes de um mesmo indivíduo. 
Como há diversos modelos que foram significativos para o problema a ser ajustado é necessário escolher um que forneça os melhores resultados possíveis e bem estimados. O teste que ajuda na escolha entre os efeitos fixos e os efeitos aleatórios utilizado nesse trabalho foi o teste de Hausman que compara as estimativas de efeitos aleatórios com as de efeitos fixos. Diferenças significativas entre elas sugerem a inconsistência dos estimadores de efeitos aleatórios. O resultado do teste é mostrado na tabela 6 abaixo:

Fonte:

autor a partir de resultados da pesquisa

Com um nível de 5\% de significância, não se rejeita a hipótese nula de que há diferenças entre os coeficientes de efeitos aleatórios se comparado com o de efeitos fixos, ou seja, rejeita-se a hipótese de que os modelos aleatórios são consistentes. Portanto, o modelo mais apropriado ajustado escolhido foi o de efeitos fixos.

Com o modelo escolhido é necessário testar a heterocedasticidade, caso tenha a heterocedasticidade não é um problema que invalide o modelo já que os coeficientes estimados continuam sendo não viesados e consistentes mas deixam de ser eficientes. Por isso é necessário que se corrija esse problema através de estimadores das variâncias robustos a heterocedasticidade.

\begin{tabular}{l} 
xttest3 \\
Modified Wald test for groupwise heterocedasticity in fixed effect regression model \\
\hline HO: sigma (i)^2 = sigma^2for all i \\
\hline chi2(35) $=429.08$ \\
\hline Prob $>$ chi2 $=0,0000$
\end{tabular}

O teste de heterocedasticidade de Wald rejeitou a hipótese nula de que há homocedasticidade no modelo escolhido, portanto é necessário corrigi-lo. Aplicando os comandos cluster para efeitos fixos no Stata, chega-se a modelos robustos quanto a variância da heterocedasticidade.

Pode-se comparar as estimativas com e sem correção, para analisar a sensibilidade da significância dos coeficientes à quebra dos pressupostos do modelo clássico de regressão 
linear. Embora as estimativas dos erros padrão (a segunda linha de cada coeficiente da tabela 7 abaixo) são diferentes. Entretanto, na presença de heterocedasticidade as estimativas dos erros de um modelo clássico de regressão linear serão tendenciosas. O que realmente importa é que o segundo modelo é mais robusto quanto a esse problema e que as relações continuam significativas. Ou seja, nenhuma variável independente “inverteu” sua relação com a variável dependente.

Fonte: elaborado pelo autor a partir de dados da pesquisa

Pelo modelo ajustado, pode-se perceber que somente a variável PDI apresentou uma relação inversa com a variável dependente Mercado. Isso quer dizer que, quando uma empresa busca ampliar mercado, a pesquisa e desenvolvimento interno a empresa não é uma variável que auxilia no objetivo. Esse resultado é confirmado por estudos de Rocha (2015) e Dosi (2000), onde no Brasil o sistema de inovação ainda é insipiente não permitindo as empresas a buscarem inovação de forma competitiva no interior do chão de fábrica.

O estudo contribui então no entendimento do gasto das empresas industriais brasileiras com os gastos de inovação. Quando uma empresa deseja ampliar mercado através da inovação, ela o faz através de Pesquisa e Desenvolvimento Externo a Empresa, Conhecimentos Externos, e Treinamentos, não assumindo o inserindo a pesquisa dentro do chão de fábrica da empresa.

\section{Considerações Finais}

O trabalho objetivou estabelecer a relação entre os dispêndios com inovação e o 
impacto dessa atividade inovativa na ampliação de mercado dos setores industriais, para os períodos de 2000, 2003, 2005, 2008 e 2011. Os dados possuem esses gaps pela própria natureza da fonte de dados, no caso a PINTEC, feita pelo IBGE, que não é anual.

Após a aplicação de metodologias de econometria de dados em painel, ajustou-se um modelo de efeitos fixos com erro-padrão robusto. Para cada unidade adicional das variáveis o impacto na ampliação do mercado é negativo em -0,43 para o PDI e positivo em 1,32 para o PDE, 1,88 para o CE e 1,84 TT, para um nível de significância de 5\%.

A relação negativa com a Pesquisa e Desenvolvimento Interno é corroborada pela literatura onde, no Brasil, para um retorno inovativo é mais rentável que a empresa busque Pesquisa e Desenvolvimento Externo a empresa, efetuando parcerias com institutos de pesquisa, por exemplo, do que desenvolvendo pesquisa interna a empresa por acarretar em custos administrativos e gerenciais elevados.

Portanto o trabalho evidenciou que dispêndios de inovação causam impactos positivos na ampliação de mercado dos setores industriais brasileiros, com exceção da Pesquisa e Desenvolvimento Interno, no qual o impacto negativo.

Para trabalhos futuros sugere-se que continuem as análises dos demais impactos da inovação disponíveis na PINTEC com os dispêndios em inovação para mensurar o impacto dos dispêndios na manutenção do mercado e no lançamento de novos produtos e processos no mercado. Espera-se também que com a nova edição da PINTEC 2014 os modelos possam ser melhores estimados.

\section{Referências bibliográficas}

CAINELLI, Giulio; EVANGELISTA, Rinaldo; SAVONA, Maria. The impact of innovation on economic performance in services. The Service Industries Journal, v. 24, n. 1, p. 116-130, 2004.

DOSI, G. Innovation, organization and economic dynamics.Selected essays. Cheltenham: Edward Elgar Publishing, 2000.

DOSI, Giovanni; NELSON, Richard R. An introduction to evolutionary theories in economics. Journal of evolutionary economics, v. 4, n. 3, p. 153-172, 1994.

FREEMAN, Chris; SOETE, Luc. A economia da inovação industrial. Editora da UNICAMP, 2008.

KIM, Linsu. Imitation to innovation: The dynamics of Korea's technological learning. Harvard Business Press, 1997. 
ROCHA, Frederico. Qual o efeito do apoio governamental à inovação sobre o gasto empresarial em P\&D? Evidências do Brasil. Revista Brasileira de Inovação, v. 14, p. 37-60, 2015.

SBRAGIA, R. (Coord.). Inovação: como vencer esse desafio empresarial. São Paulo: Clio Editora, 2006.

TIDD, J.; BESSANT, J.; PAVITT, K. Gestão da Inovação. 3. ed. Porto Alegre: Bookman, 2008.

WOOLDRIDGE, J. M. 2002. Econometric Analysis of Cross Section and Panel Data. Cambridge, MA: MIT Press.

Santos, D. Neves, M. Análise do impacto da capacidade de inovar da indústria brasileira. In: Encontro Nacional de Engenharia de Produção-ENEGEP, 33. Associação Brasileira de Engenharia de Produção-ABEPRO, 2013. 